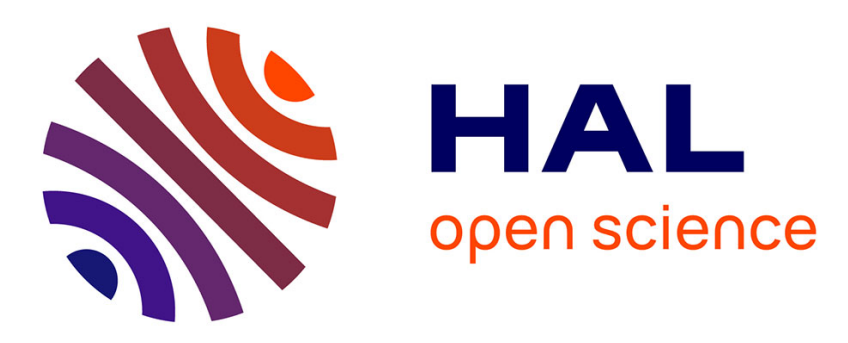

\title{
Morphological self stabilization of locomotion gaits: illustration on a few examples from bio-inspired locomotion
}

\author{
Christine Chevallereau, Frédéric Boyer, Mathieu Porez, Johan Mauny, \\ Yannick Aoustin
}

\section{To cite this version:}

Christine Chevallereau, Frédéric Boyer, Mathieu Porez, Johan Mauny, Yannick Aoustin. Morphological self stabilization of locomotion gaits: illustration on a few examples from bio-inspired locomotion. Bioinspiration and Biomimetics, 2017, 12 (4), pp.46006 - 46006. 10.1088/1748-3190/aa728f . hal01558509

\author{
HAL Id: hal-01558509 \\ https://hal.science/hal-01558509
}

Submitted on 7 Jul 2017

HAL is a multi-disciplinary open access archive for the deposit and dissemination of scientific research documents, whether they are published or not. The documents may come from teaching and research institutions in France or abroad, or from public or private research centers.
L'archive ouverte pluridisciplinaire HAL, est destinée au dépôt et à la diffusion de documents scientifiques de niveau recherche, publiés ou non, émanant des établissements d'enseignement et de recherche français ou étrangers, des laboratoires publics ou privés. 


\title{
Morphological self stabilization of locomotion gaits: Illustration on a few examples from bio-inspired locomotion
}

\author{
Christine Chevallereau and Frederic Boyer and Mathieu Porez and Johan Mauny and Yannick Aoustin,
}

\begin{abstract}
To a large extent, robotics locomotion can be viewed as cyclic motions, named gaits. Due to the high complexity of the locomotion dynamics, to find the control laws that ensure an expected gait and its stability with respect to external perturbations, is a challenging issue for feedback control. To address this issue, a promising way is to take inspiration from animals that intensively exploit the interactions of the passive degrees of freedom of their body with their physical surroundings, to outsource the high-level exteroceptive feedback control to low-level proprioceptive ones. In this case, passive interactions can ensure the most of the expected control goals. In this article, we propose a methodological framework to study the role of morphology in the design of locomotion gaits and their stability. This framework ranges from modelling to control aspects, and is illustrated through three examples from bio-inspired locomotion: a three-dimensional micro air vehicle in hovering flight, a pendular planar climber and a bipedal planar walker. In these three cases, we will see how simple considerations based on the morphology of the body can ensure the existence of passive stable gaits without requiring any highlevel control.
\end{abstract}

\section{INTRODUCTION}

Animal locomotion in almost all its forms can be thought of as cyclic motions, named gaits, which couple both the internal (shape) and external (net displacement) degrees of freedom (DoFs) of a body, to produce periodic external forces propelling the animal in space [1], [14], [22]. These gaits can then be modulated by exteroceptive feedback to perform steering maneuvers required by goal oriented tasks or reactive obstacle avoidance [40], [45], [47]. For any living organism, the locomotion gaits result from the coevolution of the nervous system in interaction with the body and the external environment. In particular, locomotion gaits are largely encoded into the animals morphology which facilitates their emergence from the passive interactions of the body with its physical surrounding. This key strategy has been successively evolved by animals along the history of life through many locomotion modes so allowing them to conquer all the environments with a wide diversity of substrates as flying in air, swimming in water, walking on the ground or climbing along complex vertical substrates to name but a few. For robotics, the understanding of these passive strategies encoded into body morphology is of crucial interest. In particular, they can be exploited for the design of a new generation of robots with controllers outsourced as far as possible from a central control unit in order to preserve its essential function: that of a decision maker. This outsourcing of gaits control to the body is an instance of the concept of Morphological Computation [31], [32] or more precisely of morphological control according to the classification proposed in [27].

To illustrate this strong concept, let us consider few locomotion modes as they are currently studied by robotics. Human walking is a complex process which remains not well understood in many points [17]. However, simple observations show that though learning to walk is a long and arduous process in the beginning of life, once learned, the act of walking across a level floor "becomes a second nature". As this is suggested by the studies of passive walking [10], [11] and derived studies [41], [43], one of the reasons of this fact, may be that the human walking gait exploits self stabilisation properties of the body in order to bypass high level control. With few exceptions, including control based on SLIP (Spring-Loaded Inverted Pendulum) model [4], this idea, which is based on morphology, remains largely unexploited in popular humanoid controllers of today [20]. More generally, many animals can use their physical surrounding to actuate some of their passive DoFs for locomotion. This is the case of systems which exploit the potential energy of the ambient gravitational field to actuate their passive external DoFs as a walker in its falling phase or a gibbon climbing in trees using pendular locomotion [3], [16], [28], [29], [39]. It can also be observed that collisions with the environment, though being often avoided in robotics, can help for stabilization of biped [6] and multilegged locomotion [37]. Other animals can use internal passive DoFs to harvest energy from their surrounding that they use for locomotion. For instance, the vortices flowing past a fish can actuate its soft caudal fin to propel its body upstream in a passive or semi-passive manner [24]. More generally, biology teaches us that contrarily to the current uses in robotics where the flexibility of bodies are considered as parasitic effects produced by the design, animals take benefit from their soft appendages to improve their locomotion performance [15], [38]. An emblematic example of that idea is that of the production of lift in hovering flight of moth, which is based on the passive twisting of their wing along their span [13].

The aim of this paper is to propose a methodological framework for adapting classical tools in control theory to morphological stabilisation of bio-inspired locomotion. In particular, one of the issues of the application of these tools to bio-inspired systems is that they are strongly dependent on the dimension of the state-space of systems and become rapidly untractable on high-dimensional systems. To overcome this issue, we propose to use the concept of 
zero-dynamics which allows reducing the dimension of the system before the stability analysis. Beyond the exposition of this framework, we will implement it on few examples from bio-inspired locomotion ranging from hovering flight of a Micro Air Vehicle (MAV) to dynamic climbing and biped walking. For all these examples, the analysis of the role of morphology in the generation and the control of their gaits will require to use concepts from dynamical systems theory as the Poincaré return map, and to exploit symmetry properties to further reduce the system dimension. In this regard, let us remark that in presence of passive internal and/or external DoFs, to seek a cyclic motion requires careful investigations to check the periodicity of the passive DoFs. Regarding stability, this is a crucial expected feature since, if a cyclic motion is not attractive (or stable) then in presence of perturbations, the gait will be lost and will require a high level control to be recovered. In the case of the soft flapping wing MAV, the size or the position of the connection point of the wing with the MAV thorax will be shown to be key parameters for flight stability. In the case of a pendular climber, the distance between the left and right hands will be shown to be a key morphological parameter to insure stability of pendulum-gaits. In the case of the walker, we will show how the morphology can be exploited in relation to its walking gait in order to state simple rules ensuring stable walking based on a disequilibrium phase occurring at each step of walking [44]. These analyses will be based on a general modeling and simulation approach also presented in the paper. From this point of view, the considered examples involve different types of contacts with the environment. For flight, the contacts are continuous and smooth forces are applied by the fluid onto the system. For walking, the contact with the environment is intermittent and ruled by a geometric constraint modeling the intersection of the foot with the ground. Climbing is an intermediate case where the contact is intermittent but the new contact can be determined freely by the activation of a section cup or magnet.

The article is structured as follows. In section II, the model of partially actuated locomotion systems is presented in the case of continuous or intermittent contacts where the system is controlled with a low level feedback controller. Then an approach allowing seeking cyclic motions is presented in section III. The notion of stable cyclic motion and the tools used to analyse the stability are presented in section IV. Three different illustrative cases are addressed. The first is the hovering flight of MAV inspired from moth studied in section $\mathrm{V}$. This case involves continuous contact and the role of the morphology of the wings on the stability is analysed. The second case is that of a climber named ROCR [39], whose limit cycles are studied in section VI. For this system, the role of the position of the hands with respect to the torso on the climbing stability is discussed. The walking of planar biped is studied in section VII. In this case, the change of support is no longer defined by arbitrary time commutations but rather by a geometric condition satisfied when the swing leg touches the ground, rather than a temporal evolution. This leads us to impose the actuated joints evolution as a function of a phasing variable instead of time. Finally, the article ends with several concluding remarks and perspectives in section VIII.

\section{Modeling OF LOCOMOTION SYSTEMS}

Before presenting our modeling methodology, we first introduce some basic definitions and notations related to locomotion systems.

\section{A. Basic definitions and notations.}

To define the configuration of our locomotion system, we attach to the ambient geometric space a fixed inertial orthonormed frame denoted by $\mathcal{F}_{e}=\left(O_{e}, s_{e}, n_{e}, a_{e}\right)$, where $a_{e}$ supports the vertical axis, and the plane $\left(O_{e}, s_{e}, n_{e}\right)$ defines an horizontal plane of reference, generally attached to the ground. The locomotion system is defined as a mobile multibody system with a tree-like structure composed of a set of $n+1$ bodies $\left\{\mathcal{B}_{0}, \mathcal{B}_{1}, \mathcal{B}_{2}, \ldots \mathcal{B}_{n}\right\}$ connected by $n$ single DoF revolute joints. One of the bodies, say $\mathcal{B}_{0}$, is arbitrarily distinguished as the reference body to which a frame named "reference frame" and denoted $\mathcal{F}_{0}=\left(O_{0}, s_{0}, n_{0}, a_{0}\right)$, is attached. The position and orientation, or pose, of $\mathcal{F}_{0}$ with respect to $\mathcal{F}_{e}$ is parameterized by the transformation $g_{0}$ in the group $G=S E(3)$ in the three-dimensional case, or $S E(2)$ in the planar case. The time-evolution of $g_{0}$ defines the rigid net motion of the locomotion system. The net velocity of the reference body is denoted $\eta_{0}$. In the general three dimensional case, it is a vector of $\mathfrak{g}=s e(3)$ defined by $\eta_{0}=g_{0}^{-1} \dot{g}_{0}=\left(V_{0}^{\top}, \Omega_{0}^{\top}\right)^{\top}$, where $V_{0}$ and $\Omega_{0}$ are the inertial linear and angular velocities of $\mathcal{F}_{0}$ respectively, both being expressed in $\mathcal{F}_{0}$. The space of internal DoFs or "shape space" is denoted $\mathcal{S}$ and parameterized by the vector of joint angles $r=\left(r_{1}, \ldots, r_{n}\right)^{\top}$. In all the following, the internal variables can be actuated or not and $\mathcal{S}$ is defined as the product $\mathcal{S}=\mathcal{S}_{a} \times \mathcal{S}_{p}$, where the first factor denotes the actuated shape subspace and $\mathcal{S}_{p}$ is its passive counterpart. The $n_{a}$-dimensional subspace $\mathcal{S}_{a}$ is parameterized by the $r_{a}$-vector of actuated joint angles which belongs to $\mathbb{R}^{n_{a}}$ while the $\left(n-n_{a}=n_{p}\right)$-vector of non actuated angles, gathered in $r_{p} \in \mathbb{R}^{n_{p}}$, parameterizes $\mathcal{S}_{p}$. As regards the control torques exerted inside the locomotion system through its joints, they are gathered in the vector $\tau=\left(\tau_{1}, \ldots, \tau_{n}\right)^{\top}=$ $\left(0_{n_{p}}^{\top}, \tau_{a}^{\top}\right)^{\top}$, where $\tau_{a}$ denotes the torque-vector applied on the actuated joints. Let us note that though the passive joints are not actuated, they can be subject to passive torques modeling the joint rheology through known functions of the current state (e.g. by lumped spring and/or damper). In the following, $q_{p}=\left(g_{0}^{\top}, r_{p}^{\top}\right)^{\top}$ parameterizes the passive configuration space $G \times \mathcal{S}_{p}$. In the same way, $\dot{q}_{p}=\left(\eta_{0}^{\top}, \dot{r}_{p}^{\top}\right)^{\top}$ parameterizes the space of passive velocities in $\mathfrak{g} \times \mathbb{R}^{n_{p}}$, and $x=\left(q_{p}^{\top}, q_{a}^{\top}, \dot{q}_{p}^{\top}, \dot{q}_{a}^{\top}\right)^{\top}$ defines the full state vector. Finally we define the space of extended passive states, noted $\mathcal{V}^{+}$, as the space of $z=\left(q_{p}^{\top}, \dot{q}_{p}^{\top}, t\right)^{\top}$ with $t \in \mathbb{R}^{+}$denoting the time. With these notations in hand, we can state the locomotion dynamics modelling problem we address in this article. 


\section{B. Statement of the Low-level controlled locomotion dynam-} ics modelling problem

In the following, we consider the case of holonomic systems possibly subject to hard intermittent external forces. A natural way to address locomotion control consists of a hierarchical approach structured in three levels. The first lowest level consists of local controllers that steer $r_{a}$ toward a prescribed time-evolution $t \mapsto r_{a}^{d}(t)$. This time-evolution is prescribed by an average level able to calculate the cyclic motion patterns, or gaits. These gaits are themselves modulated by the highest, goal-oriented, control level based on exteroceptive feedback. In this article, we focus our attention on the average level, i.e., we consider the system to be directly controlled by a given actuated motion $t \mapsto r_{a}^{d}(t)$ and will study the conditions of existence of periodic passive (net and shape) motions or "limit cycles" as well as the conditions of their stability. In this context, one can model the dynamics of a holonomic system subject to smooth and/or hard intermittent contacts through the following hybrid statespace equations:

$$
\left\{\begin{array}{llll}
\dot{z} & =f(z) & z & \notin S \\
z_{+} & =f_{+}(z) & z & \in S
\end{array} .\right.
$$

In control theory, (1) defines the hybrid zero dynamics of the input-output map $\tau_{a} \mapsto r_{a}$ [49]. In our context, these zero dynamics stand for the dynamics of the system when the actuated joints time evolution is perfectly specified through our low-level torque control. As a result, the control torques do not appear in (1) since they are used to enforce $r_{a}=r_{a}^{d}(t)$. Going into details, in (1), we introduced the following notations and definitions:

- $S \subset \mathcal{V}^{+}$stands for a submanifold of dimension $\operatorname{dim}\left(\mathcal{V}^{+}\right)-$ 1 which models the conditions that the extended state satisfies when the impacts occur. For walking, $S$ stands for switching manifold, defined by:

$$
S:=\left\{z \mid p_{s}\left(q_{p}, t\right)=0, \dot{p}_{s}<0\right\},
$$

where $p_{s}$ is the vertical position of the swing foot in the ground frame $\mathcal{F}_{e}$ and the time-dependency is introduced by $r_{a}=r_{a}^{d}(t)$. A step of the robot is a solution of (1) that starts with the robot in $S$, ends in $S$ with the configurations of the legs swapped, and contains only one impact event. Walking or climbing is a sequence of steps. For climbing, the choice of transition is more arbitrary, since the attachment to the wall is arbitrarily prescribed by magnet control. Thus, the condition of switching is a simple temporal condition:

$$
S:=\{z \mid t=k T\},
$$

where $T$ denotes a fixed time-period. Though for flapping flight the motion is continuous along time, the above hybrid formulation will be used and the stability analysis of hovering flight will be carried out with the section $S$ defined by (3) where $T$ will be the time-period of the actuated motion.
- $f$ denotes a vector field which lies on $\mathcal{V}^{+}-S$, while $f_{+}$ is a map from $S$ to the full state space. $f$ and $f_{+}$govern the continuous dynamics between two transitions, and the discontinuous ones when $z$ crosses $S$, respectively. In the following, these two fields will be of the following form:

$$
f(z)=\left(\begin{array}{c}
\dot{q}_{p} \\
M^{-1} F \\
1
\end{array}\right), f_{+}(z)=\left(\begin{array}{c}
\phi\left(q_{p}\right) \\
\Delta \dot{q}_{p} \\
0
\end{array}\right),
$$

where the third row of $f$ simply reads $\dot{t}=1$ and allows changing a time-varying system into an autonomous system for which the clock is reset at step transition by the third row of $f_{+}$. In the above expressions of $f$ and $f_{+}$, we introduced the following notations. $M$ is the inertia matrix of the locomotor system while $F$ is the vector of the inertia, internal passive, and external generalized forces, the latter being noted $F_{\text {ext }}$. Note that all these matrices are defined on the configuration space $G \times \mathbb{R}^{n_{p}}$ of the system and that they can depend on time through the time evolution $t \mapsto r_{a}^{d}(t)$ and its first and second time-derivatives. In (4), $\Delta$ is a dimensionless transition-matrix modelling how the passive velocities suddenly change when the system crosses $S$, while $\phi$ is a diffeomorphism of $\mathbb{R}^{n_{p}} \rightarrow \mathbb{R}^{n_{p}}$ which allows re-parameterizing the configuration between two steps. In $f$ and $f_{+}$, the contacts that are responsible of the net motions are modelled through $F_{\text {ext }}$ and $\Delta$ respectively. Since they play a central role in locomotion modelling we consider below these two models in the cases we will study latter: a flying MAV along with our walker and climber.

1) Modelling of smooth external contacts: In the case of soft contacts, the model of the external forces $F_{\text {ext }}$ exerted by the surrounding medium on the system can be completely defined as a smooth function of the current state of the system and possibly, of its accelerations through added mass forces ${ }^{1}$. For instance, in the case of the flight (see figure 1) of a Micro Air Vehicle (MAV) with soft wings inspired from flying insects, the aerodynamic forces is in essence a function of the current state of a complex flow of air surrounding the flapping wings. In general, due to the complex topology of such a flow, the model of contact requires to integrate its Navier-Stokes equations at each time step of the simulation what is far from being achievable for complex geometries and at any Reynolds number. However, for prototyping and control-oriented applications, it is preferable to use a simplified nonlinear analytical model based on experimental observations as the model of Dickinson [13]. This model considers two types of aerodynamic forces: the quasi-steady forces whose time dependency is due to the body kinematics and the added mass forces due to the fluid accelerations. These forces exerted along the wing are modelled through their cross-sectional density along the wings $\operatorname{span}^{2}$. Going

\footnotetext{
${ }^{1}$ In this case, the added mass forces can be factorized in terms of some fluid inertia, that are added to the body inertia $M$ in the general form of $f$ which is so preserved.

${ }^{2}$ In aerodynamics, the span (respectively, the chord) of an aerofoil refers to the imaginary straight line joining the wing basis (embedded in the body) and its tip (respectively, the leading and the trailing edge).
} 
further into details, the quasi-steady forces are composed of the lift and drag forces applied on the center of pressure of each cross-section, or "blade", of the wing which are function of the square of the pressure center speed, the incidence angle of the cross-section with respect to the air flow speed, the mass density of air, and a set of empirical coefficients obtained from experiments [13]. Regarding the added mass forces, they can be derived from a kinetic momentum balance applied to the fluid which laterally bounds a wing section [33]. Finally, let us point out that since the systems subject to smooth external contacts do not experience discontinuity of their state, their vector field $f_{+}$of (1), merely stands for the identity.

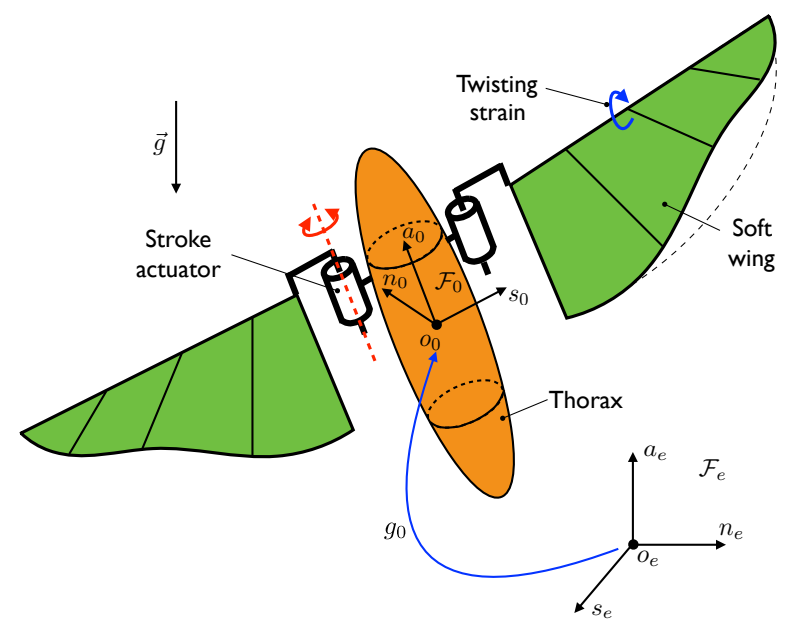

Fig. 1: Schematic view of a soft micro air vehicle bioinspired from the sphinx moth Manduca sexta in a unbounded 3-D space filled of initially quiescent air. The system is composed of a rigid thorax and two soft wings undergoing twisting deformations distributed along their leading edge. The thorax and the wings are connected through two revolute joints (one per wing). These joints are actuated and generate the typical stroke observed in the flying insect.

2) Modelling of non-smooth external contact: When dealing with terrestrial locomotion as walking, running, climbing or crawling, the contacts can be assumed to be rigid or "hard", an assumption which allows avoiding a model of penetration into the ground. In this case, the contacts can be modeled through simple kinematic constraints, deduced from the assumption that the relative velocities between the system and its solid substrate are zero in the points of contact. This is notably the case of nonholonomic wheeled systems or that of a walking and climbing robot. The first type of systems is governed by hard persistent contacts while the second is subject to hard intermittent contacts. In this second case, which is more directly related to the examples addressed in this article, the holonomic constraints can be used to reduce the number of independent variables that describe the configuration and situation of the robot. For instance, in our examples, the group of net displacements $G=S E(3)$ can be replaced by
$S O(2)$, where $S O(2)$ now stands for the rotation around the left or the right foot (respectively, wrist) of the walker (respectively, of the climber). Going further into details, for the walker (respectively, for the climber), $r_{a}$ includes the knee and hip angles (respect. the pendulum root angle), while for both systems, $q_{p}=g_{0} \in S O(2)$ stands for the rotation of the shin (respectively, of the wrist) in the sagittal plane of the walker (respect., the frontal plane of the climber). In the case of the walker, for the aim of control developed in section VII, it is relevant to introduce a further passive angular variable along one step leading to a re-parametrization of the model. In such a case, we can define a diffeomorphism: $\left(g_{0}, r_{a}\right) \mapsto\left(\theta, r_{a}\right)$, where $\theta$ represents the angle between the vertical axis and the line linking the contact point and the stance hip (see figure 2). In the case of human-like walking gaits, $\theta$ has a monotonic evolution with respect to time, a property which is crucial for control purposes with virtual constraints [48]. In this context, we shall denote $q_{p}=\theta$ in both examples. However, for the climber, $\theta$ denotes the natural angle (given by the mechanical modeling) between the vertical and the line linking the two hands. The contact is intermittent thus the
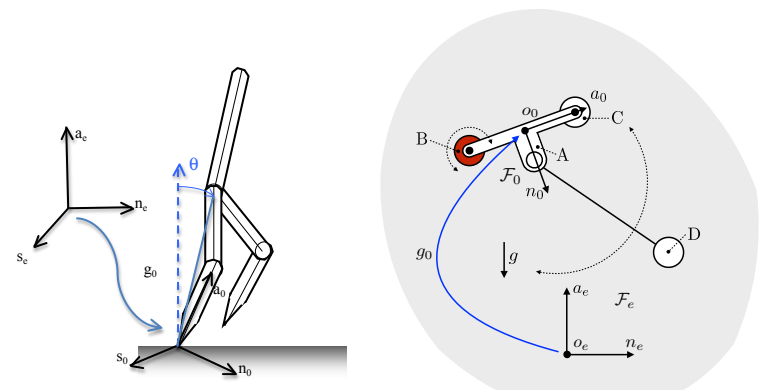

Fig. 2: Schematic view of the planar biped and of the climber, the contact with the ground is a passive rotation.

dynamics of transitions are modeled though impacts that are assumed to be plastic and to have an infinitely short duration, fully encoded in $\Delta$ of (4), a matrix which can be easily deduced following a classical process changing continuous into discrete dynamics [21]. In the cases we will address in this article, at each transition, our climbing and walking robot instantaneously changes of contact point (change of leg for the walker and of arm for the climber as drawn in figure 2) by switching between two different sets of holonomic constraints. In these conditions, the dynamic balance relating the kinetic momentums and force impulses stands for the angular momentum conservation law and $\Delta$ models the jump of velocities. Each of these sets of constraints models a revolute joint connecting a wrist of the climber and a foot of the walker to the substrate. The reduction of net displacement (group) variables, which is allowed by the holonomic character of the constraints, imposes a change of parametrization (underlaid by a shift of reference frame from one contact point to the other) at each transition. In (4), this change of parametrization, 
which applies to $q_{p}=\left(g_{0}^{\top}, r_{p}^{\top}\right)^{\top}$, is accounted by both the diffeomorphism $\phi$, and the transition-matrix $\Delta$.

Remark 1: It has to be noted that the model (1) needs to be invariant under the reset map (2) or (3). In the case of flapping flight this condition is trivially ensured since the system has no impacts. In the two other cases, the first equation of (1) must be satisfied just after the impact. In the case of planar walking, this can be ensured by an appropriate choice of the reference $r_{a}^{d}$ [48]. In the case of 3D walking, a deadbeat control must be added to be able to perform this condition [8]. In the case of climbing robot, it is assumed that the actuator is able to produce impulsive force in order that the reference $r_{a}^{d}(t)$ will be perfectly followed through impact. In reality, such impulsive torques cannot be provided by the actuators but are filtered by the local dynamics of mechanical joints.

Remark 2: The hybrid model (1) which describes the different phases (single support, impact), has been written in a form where the actuated variables are controlled in position with a perfect tracking, i.e., in a form where the inputs are the desired trajectory of the actuated variables, and the outputs are the accelerations of the passive ones. Though the above form is a simplification of the original model in which the state dimension is reduced to the number of degrees of under actuation (the passive reduced DoFs), such a form is well suited to systems with variables ruled by passive physical laws describing the effect of flexibilities or gravity. In fact, such a reduced model contains all the dynamic characteristics of the original system since the simplification of the model only lies in the fact that the tracking error $r_{a}^{d}-r_{a}$ ensured by the low-level control law is neglected. Furthermore, when investigating the effects of morphology, this approximation is enough accurate and provides richer insights than other approaches based on the model of pendulum, as those often used in the study of walking locomotion when an humanoid is approximated by an inverted pendulum with a lumped mass.

\section{CYCLIC MOTION OF LOCOMOTION SYSTEMS}

Our first goal is to seek cyclic motions, or gaits, of our locomotion systems. In this regard, note that while a cyclic evolution of the internal actuated variables can be trivially performed with the low-level controllers, a periodic evolution of the non-actuated (passive) variables is far from being so easily achievable. In this section, this general issue is addressed both for continuous and discontinuous contacts. In both cases, the motion of actuated variables will be defined as a $T$-periodic time-function $\left(r_{a}^{d}(t)=r_{a}^{d}(t+T)\right)$. For piecewise continuous systems as the walker and the climber, each cycle is naturally defined by two successive satisfactions of the commutation conditions (2) and (3). In the case of continuous contacts, a cycle is only specified by the $T$-periodicity of $r_{a}^{d}$ and no physical consideration allows distinguishing some particular starting and ending times of the cycles.

\section{A. Seeking the cyclic motions of locomotion systems}

The Poincaré return map is a classical tool for studying the cyclic motions of dynamic systems [18], [30]. In our case, we apply this tool to the following dynamics whose form is enough general to include all the three examples studied:

$$
\left\{\begin{array}{llrl}
r_{a} & =r_{a}^{d}\left(q_{p}, t\right) & z \notin S \\
\ddot{q}_{p} & =f_{\text {zero }}\left(q_{p}, \dot{q}_{p}, t\right) & z \notin S \\
z^{+} & =\Delta\left(z^{-}\right) & z & \\
d t / d t & =1, & &
\end{array}\right.
$$

where the third equation becomes $z^{+}=z^{-}$for a continuous model of contact. In (5), we recognize the passive zerodynamics of the low-level controlled locomotion dynamics of (1), except that the first two equations express the fact that the controlled motion of the actuated variables can be a function of the passive variables (as it will be proposed in section VII in the case of our walker) or of time (in the case of our climber and our hovering MAV) [12]. In this later case, since the state transitions depend on time, the system is changed into an autonomous one by using the augmented state $z=\left(q_{p}^{\top}, \dot{q}_{p}^{\top}, t\right)^{\top}$ with the trivial equation $d t / d t=1$ appearing in (5). In this context, we define a Poincaré section in the augmented passive state space $\mathcal{V}^{+}$, as a set $\left.S_{p}=z \mid h(z)=0\right\}$, with $h$ a real valued function defining implicitly the Poincaré section. We take a point $Z_{k}=\left(q_{p}^{\top}, \dot{q}_{p}^{\top}, 0\right)^{\top} \in S_{p}$, and consider it as an initial condition for (5) that we numerically integrate until recrossing $S_{p}$. This crossing point naturally defines the image of a return map $P: S_{p} \rightarrow S_{p}$. Thus the Poincaré return map $Z_{k+1}=P\left(Z_{k}\right)$ can be defined numerically and its fixed points define the periodic orbits of the underlying system $P\left(Z^{*}\right)-Z^{*}=0$, i.e., the limit cycles of the locomotion system governed by (5).

Remark 3: In the case of our planar walker, the intermittent contacts are defined by a geometric condition (2) where the time does not appear explicitly. Thus, by prescribing a set of virtual constraints through control, one can reexpress the desired evolution of actuated variables in terms of the current configuration of the locomotion system $q_{p}$ only [5], [23], [48]. This allows one to completely remove the time from the formulation (5) and to define the Poincare return map directly by the crossing condition (2) which stands for its Poincaré section.

Remark 4: In all cases, the expected periodicity of the unactuated (passive) variables can be prescribed on one single cycle as the following condition:

$$
P\left(\left(\begin{array}{c}
q_{p}(0) \\
\dot{q}_{p}(0) \\
0
\end{array}\right)\right)-\left(\begin{array}{c}
q_{p}(0) \\
\dot{q}_{p}(0) \\
0
\end{array}\right)=\left(\begin{array}{c}
0 \\
0 \\
T
\end{array}\right)
$$

where let us note that this condition (6) is directly usable for any system explicitly dependent on the time, as this is the case of our climber and the MAV. For the walker, since the time does not appear in the cyclicity condition, the last equation can be removed from (6), or it can be 
used to obtain cyclic motion with a given period. The first equation of (6) generally contains components related to position and orientation. In all the following, the motions studied are limited to cases where the same orientation of the reference body is expected at the end of each cycle. Regarding position, if as in hovering flight, an in-place motion is desired, the position must be unchanged as it is stated by the first equation of (6). In other cases, as for walking or climbing, the desired displacement will be $\langle v\rangle T$ where $\langle v\rangle$ is the average linear velocity of the origin of frame $\mathcal{F}_{0}$ in $\mathcal{F}_{e}$, and thus non-zero values will replace some of the zero RHS members of (6). To seek the cycles solution of (6), we consider $q_{p}(0), \dot{q}_{p}(0)$, as free variables of the problem, which turns into a boundary value problem.

Remark 5: In practice, the boundary value problem (6) is addressed with numerical optimisation techniques. Starting from a set of initial value $q_{p}(0), \dot{q}_{p}(0)$ and with a prescribed $r_{a}^{d}$, the numerical integration of (5) over one cycle, allows one to compute the left-hand-side of (6). Then, if the desired value given in the right-hand-side of (6) is obtained by the integration, the desired cyclic motion is found and the problem is solved. In other cases, new values for $q_{p}(0), \dot{q}_{p}(0)$, are fed back by the algorithm according to an iterative strategy. To apply such a general algorithmic process it is helpful to adapt it to each considered locomotion issue.

\section{B. Exploitation of the symmetries}

It is helpful to reduce the dimension of the original boundary value problem by exploiting the symmetry properties of the system. A first source of symmetry is offered by the resting morphology of bio-inspired locomotion systems which generally enjoys bilateral (right/left) symmetry leading to a first reduction as soon as one considers actuated motions which preserve this symmetry. For instance, we will consider symmetric motions for the left and right wings for flight, or symmetric motions of the left and right legs (respectively, of arms) for our walker (respectively, for our climber). With these restrictions, the periodical displacements of the robot belong to the symmetry plan of its geometry (e.g. the sagittal plane of our MAV) and some of the expected conditions of (6) are defacto satisfied for any reduced initial state complying with these symmetries. A second source of symmetry is due to the external forces applied to the locomotion systems. This results in the fact that the dynamic models of our systems are invariant by a change of the origin of $\mathcal{F}_{e}$ and a rotation around the vertical inertial axis. Thus, it is expected that some of the variables of $g_{0}(0)$ are not affected by the motion of the robot, or equivalently can be chosen arbitrarily in the boundary value problem statement. Often, the number of constraints (6) becomes larger than that of the variables $q_{p}(0), \dot{q}_{p}(0)$ that actually affect the zero-dynamics. As a result, the addressed optimization problem may have no solution for any arbitrary evolution $r_{a}^{d}$. To circumvent this issue, $r_{a}^{d}$ can be written as a parametric function whose free parameters are considered as optimization variables of the algorithm in the same way as the initial passive state.

\section{STABility ANALYSis}

When a cyclic motion has been found, the question of its stability naturally arises. It can be formulated as follows. What happens if the state of the locomotion robot is close to the limit cycle but not exactly on it? Does its motion along the system dynamics converge to the cyclic motion or does it deviate from it? To be addressed, this further issue requires to analyse the stability of the Poincare return map previously defined. Going further into the details, the eigenvalues of the Jacobian of the Poincaré map evaluated around the fixed point characterize the local stability of the cyclic motion. For a state of the robot in the Poincare section close to $Z^{*}$ we have: $Z_{k+1}-Z^{*}=P\left(Z_{k}\right)-Z^{*} \simeq J\left(Z^{*}\right)\left(Z_{k}-Z^{*}\right)$, with $J$ the Jacobian of $P$ whose eigenvalues feature its stability. More precisely, if the magnitudes of all the eigenvalues of $J$ are strictly less than one, then the limit cycle is asymptotically stable. In our context, that means that the stability of the cyclic gaits can be ensured with no further control except the low level control of the actuated DoFs imposing the convergence of $r_{a}-r_{a}^{d}\left(q_{p}, t\right)$ to zero. In other words, the stability is ensured just by exploiting the morphology of the body and of the gaits, both encoded into (1). In cases where such a semi-passive stability cannot be obtained, i.e. if the limit cycle remains unstable, we need to stabilize it using high level control based approaches [8]. In the following, we will show on several examples (a soft MAV, a climber and a walker) how the morphological characteristics of a locomotion system can be exploited in relation to its gaits, to perform stable cyclic motions with no need of high level controllers.

\section{Hovering FLIGHT OF A SOFT WINGS MAV}

The tools presented in this paper have already been extensively used for walking and climbing robot, but to our knowledge, their use in the case of soft systems is quite new and particularly adapted. It will be shown in the case of mechanical system involving flexible joints and very few actuated joints, that the above stability analysis is an efficient tool for ensuring morphological self stabilization of all the passive dofs including the flexible joints.

Moths as the Sphyngidae Manduca Sexta use the spanwise twisting of their wings to produce the lift required by hovering flight [13]. This process lies on a passive coordination of the actuated periodic joints at the wings root (the connection points to the thorax), and the wings deformation. In the following, we shall see to which extent the stability of the hovering flight (modeled as a limit cycle) can be performed in a passive way depending on some morphological features of the MAV such as the torsion stiffness of its wings, and other geometric characteristics. This is of crucial interest for bio-robotics, since if such a limit cycle can be naturally stabilized by morphology, hovering flight can be performed with simple open loop control (or local feedback control of the two actuated wings roots). Due to the approximations introduced by our idealized models, 
note that our purpose is not to recover the solutions invented by nature but rather to illustrate the role of morphology in stability with our general method. However, the starting design is directly inspired of the moth manduca sexta [19] (see [34] for details).

\section{A. Parametrization and low level control}

The soft wing MAV considered in this study is presented in figure 3. The model satisfies a right/left symmetry thus only half of the robot is shown. It can be noted that only the flapping joints, parameterized by $r_{a r}$ for the right wing, and $r_{a l}$ for the left wing, are actuated where the notation $l$ and $r$ denote the right and left wings. Observing moth in

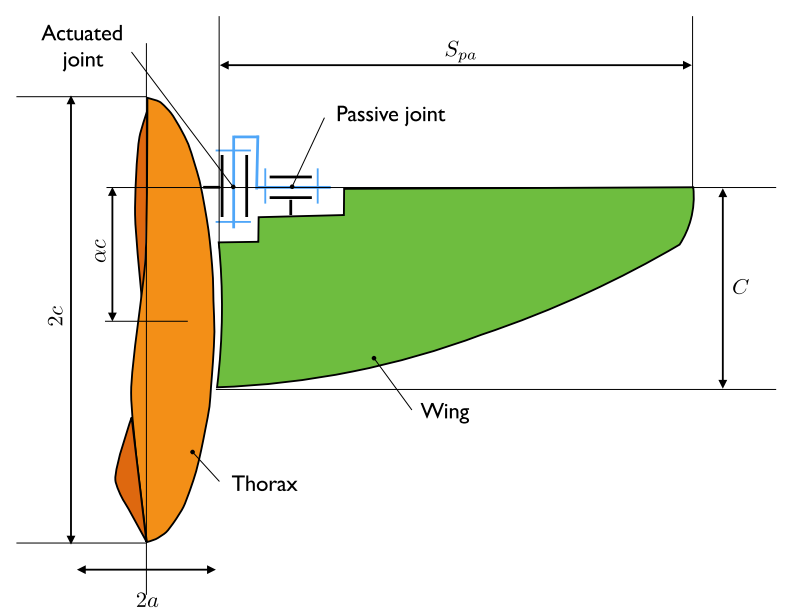

Fig. 3: Half model of the soft wing MAV. $S_{p a}$ and $C$ denote the span of the wing and its maximal chord respectively.

hovering flight [50], and to preserve the right/left symmetry, the periodic motion of our two flapping wings is imposed through a periodic symmetric sinusoidal motion of the typical form:

$$
\begin{aligned}
& r_{a r}=A \cos (w t), \\
& r_{a l}=-r_{a r} .
\end{aligned}
$$

Practically, such a motion can be for instance produced by one or two actuators on each wing controlled by a Central Pattern Generator or through a four-bar mechanical system [25].

The passive internal variables are the joint variables of the lumped twist springs along the leading edge wings. They are gathered in the two vectors $r_{p r}$ and $r_{p l}$ related to the right and the left wings respectively. The passive external variables consist of the pose $g_{0}=\left(R_{0}, P_{0}\right)$ of the MAV reference body (named the thorax) in the inertial frame $\mathcal{F}_{e}$. The orientation of the MAV is parameterized as $R_{0}=\operatorname{Rot}(a, \psi) \operatorname{Rot}(s, \theta) \operatorname{Rot}(n, \phi)$, where $\psi, \theta, \phi$ denote the yaw, pitch and roll angles respectively ${ }^{3}$. As a result, the state of the passive variables is defined by:

$$
z_{p} \triangleq\left(q_{p}^{T}, \dot{q}_{p}^{T}\right)^{T}=\left(P_{0}, \psi, \theta, \phi, \eta_{0}, r_{p}, \dot{r}_{p}\right)^{T} .
$$

${ }^{3}$ This choice of representation of orientation with three independent variables admits singularities naturally avoided by the expected orientation of the MAV. The axis $s_{0}, n_{0}, a_{0}$ are shown on figure 1

\section{B. Hovering flight}

Once the motion of the actuated variables is fixed through (7), the motion of the MAV is entirely defined by the initial value of its passive (internal and external) state variables and its dynamic model [33]. Cyclic motion can be obtained through the numerical resolution of the boundaryvalue problem defined in section (III). To that end, we need to invoke physical properties and symmetries of the system. First let us remark that for an arbitrary flapping motion (7) defined by $A$ and $w$, the cyclic motion cannot be found by solely varying the initial state of the passive variables (8). This can be physically explained by remarking that a hovering motion exists only if along each wing's beat, the lift produced by aerodynamics exactly balances the weight of the MAV. Then, since the lift can be preserved by simultaneously decreasing the wing beats amplitude and increasing its frequency; once the beat frequency is chosen, one cyclic motion can only be found if the stroke amplitude $A$, is considered as a free variable. Second, since the external forces exerted on the MAV are only due to gravity and to the aerodynamic forces, the dynamic model is invariant with respect to the position and the yaw orientation of the thorax in the fixed frame. Thus, the four first variables $\left(P_{0}, \psi\right)$ of $z_{p}$, can be chosen arbitrarily. Moreover, since the resting geometry of the MAV and the actuated time evolution $r_{a}^{d}(t)$ of (7) are symmetric with respect to the body transverse plane $\left(n_{0}, a_{0}\right)$, the cyclic motions we seek are also symmetric in this regards, and satisfy $V_{0 y}=0, \Omega_{0 x}=0, \Omega_{0 z}=0$, $\phi=0, r_{p l}=-r_{p r}, \dot{r}_{p l}=-\dot{r}_{p r}$. Finally, based on all these remarks, the limit cycles of hovering flight are sought by the shooting algorithm of section III, using the reduced set of optimization variables: $x_{o p t}=\left\{\theta, V_{0 x}, V_{0 z}, \Omega_{0 y}, r_{p r}, \dot{r}_{p r}, A\right\}$ with $w$ chosen arbitrarily $(w=2 \pi / T)$. For the initial

TABLE I: Initial values of the design parameters of the MAV of figure 1. $S_{p a}$ is the span of the wing. $C$ is the maximal chord (the wing having the shape of a quarter of an ellipse with a small axis equal to the half of $C$ ). $n_{b}$ is the number of blades, here one, connected to the rigid leading edge through a torsion spring of stiffness $K_{e}$ and damping $K_{v} . \alpha$ measures the height of the connection point of the actuated joints above the ellipsoidal body's Center of Mass (CoM).

\begin{tabular}{|l|c||l|c|}
\hline Parameter & Value & Parameter & Value \\
\hline$n_{b}$ & 1 & $\alpha$ & 0.5 \\
$S_{p a}$ & $70 \times 10^{-3} \mathrm{~m}$ & $K_{e}$ & $3 \times 10^{-3} \mathrm{Nm} / \mathrm{rad}$ \\
$C$ & $31.5 \times 10^{-3} \mathrm{~m}$ & $K_{v}$ & $1 \times 10^{-6} \mathrm{Nms} / \mathrm{rad}$ \\
\hline
\end{tabular}

design of the MAV defined in table I [33], and one passive (twisting) joint per wing, cyclic motions have been found for a wing-beat frequency ranging from $7 \mathrm{~Hz}$ to $43 \mathrm{~Hz}$. Different hovering flights controlled with the beating law (7), are illustrated on figure 4, which shows the needed amplitude expressed as a function of the frequency. For a wing-beat frequency of $25 \mathrm{~Hz}$, the optimization process ensures a periodic sustentation against gravity for amplitude of $0.945 \mathrm{rad}$ or $54^{\circ}$ as shown in figure 4. For the sphinx Manduca Sexta, the wing beat frequency is around $25 \mathrm{~Hz}$, 


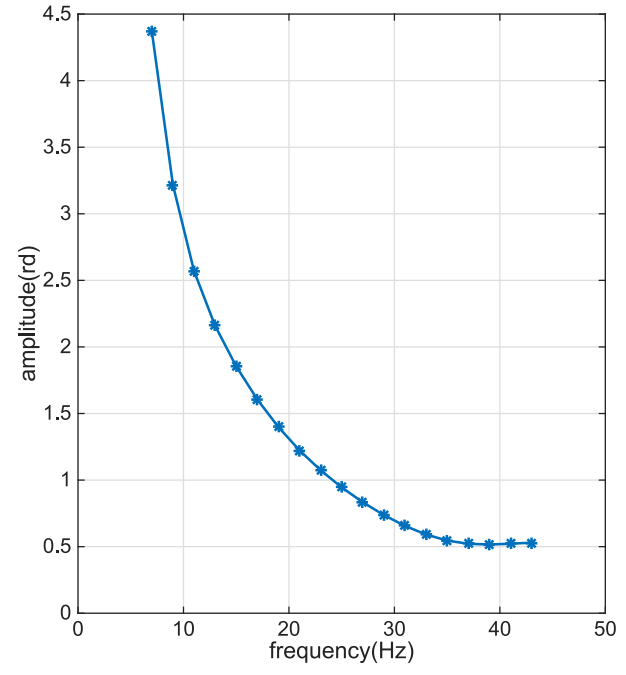

Fig. 4: Amplitude versus frequency of the actuated flapping motions.

and the flapping amplitude is around $60^{\circ}$ [50]. Due to aerodynamics, the actuated flapping motion creates a passive twist of the wing and a net lift on the thorax. Several views of the MAVs during one cycle of hovering flight are shown in figure 5. Note that even with a sinusoidal flapping motion, the non linearities of the dynamic model and forces produce a motion of twist that may be non-sinusoidal.

\section{Stability of hovering flight}

We have seen that for the proposed design, cyclic motions exist and that these motions are qualitatively close to the motions observed in moth with a large twist motion and a motion of the thorax aligned in the plane $\left(n_{0}, a_{0}\right)$. A further issue is to determine if these cyclic motions are stable or not. Even with only one twisting passive joint per wing, the number of passive state is high, the dimension of $z_{p}$ being equal to 16 . Thus 16 eigenvalues of the Poincaré map have to be calculated. Fortunately, the symmetry properties of the model can be exploited to rewrite the Poincare map (and its Jacobian) in a new chart of the state space (i.e. a new set of state variables deduced from the original one through a diffeomorphism), in which the Jacobian presents some decoupling properties whose exploitation greatly simplifies the analysis of stability. This diffeomorphism is defined by the following relations where $y_{p}$ denotes the new state vector:

$$
\begin{aligned}
& y_{p}=\left[y_{p 1}, y_{p 2}, y_{p 3}\right] \text { with } \\
& y_{p 1}=\left[P_{0}^{*}, \psi^{*}\right] \\
& y_{p 2}=\left[V_{0 y}, \Omega_{0 x}, \Omega_{0 z}, \phi, \frac{r_{p r}+r_{p l}}{\sqrt{2}}, \frac{\dot{r}_{p r}+\dot{r}_{p l}}{\sqrt{2}}\right] \\
& y_{p 3}=\left[V_{0 x}, V_{0 z}, \Omega_{0 y}, \theta, \frac{r_{p r}-r_{p l}}{\sqrt{2}}, \frac{\dot{r}_{p r}-\dot{r}_{p l}}{\sqrt{2}}\right]
\end{aligned}
$$

In (9), $y_{p 1}$ gathers the four variables that have no effect on the flight, while $y_{p 2}$ gathers the six variables that correspond to asymmetric motions. Thus, for a symmetric flight, we have $y_{p 2}=0$. The last six variables gathered in $y_{p 3}$ are

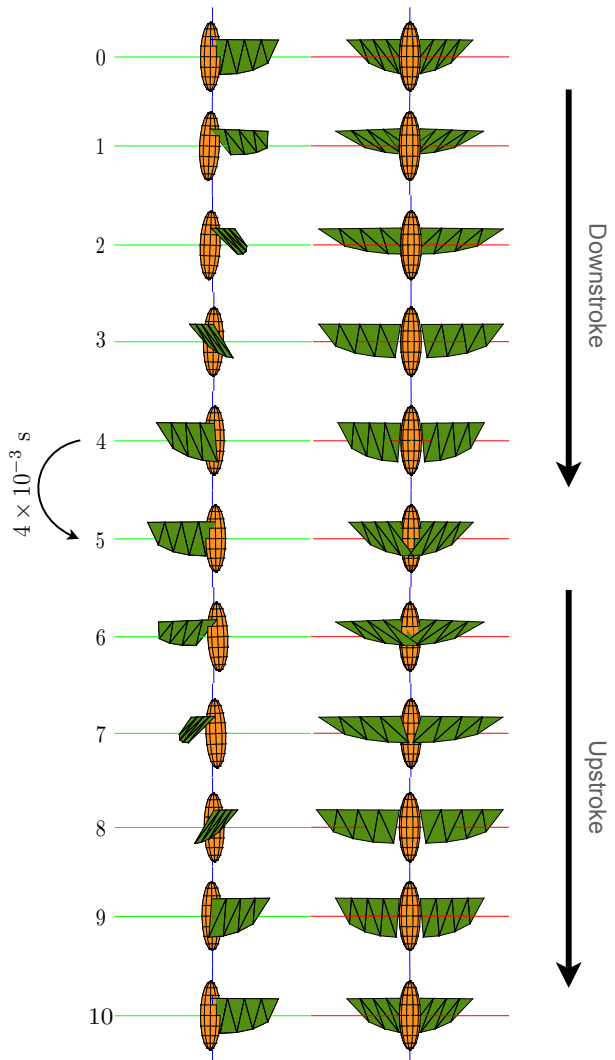

Fig. 5: Snapshots of the MAV over one period of hovering flight at $25 \mathrm{~Hz}$.

independent of $y_{p 1}$ and $y_{p 2}$ and characterize our symmetric flight motions. An orthogonal transformation matrix allows changing the representation of the state from $z_{p}$ to $y_{p}$. Since the transformation matrix between the two representations is orthogonal, the eigenvalues of the Jacobian of the Poincare map are unchanged when shifting from the $x_{p}$ to the $y_{p}$ state representation. In terms of these new $y_{p}$-variables, the Jacobian matrix can be written as:

$$
J=\left(\begin{array}{ccc}
I_{4} & C & D \\
0_{6,4} & A & 0_{6,6} \\
0_{6,4} & 0_{6,6} & B
\end{array}\right) .
$$

Hence, the eigenvalues of $J$ are the eigenvalues of $I_{4}$, the eigenvalues of $A$, and those of $B$. The four unit eigenvalues stand for the position of the thorax and the yaw angle. Since the model is invariant with respect to these parameters, an error on these values cannot converge to zero. In other terms, if a cyclic motion exists for one position and one yaw angle, the same cyclic motion exists for any other position and yaw angle. It is also worth noting that there is no coupling effect between the variables of $y_{p 2}$ and those of $y_{p 3}$. This decoupling property allows one to separate the eigenvalues associated to the errors on $y_{p 2}$ and $y_{p 3}$, and to characterize more easily the influence of a given design on the stability of the hovering flight. 


\section{Stability analysis of the initial design}

In figure 6, the norm of the largest eigenvalues of matrices $A$ and $B$ are presented for different beat-wing frequencies of the hovering flight of the MAV with design of table I. From these plots, it can be inferred that for a wing-beat frequency higher than $18 \mathrm{~Hz}$, the maximal norm of the eigenvalues of $A$ is less than 1 , so ensuring stability and the convergence of $y_{p 2}$ toward 0 . However the maximal norm of the eigenvalues of matrix $B$ being higher than 1, the stability cannot be satisfied in this case, and if an error on $y_{p 3}$ appears, this error will increase and the motion will not converge toward the desired hovering flight. To face this natural instability, we will explore further how the design of the MAV of table I can be modified to naturally ensure the limit cycle to be attractive.

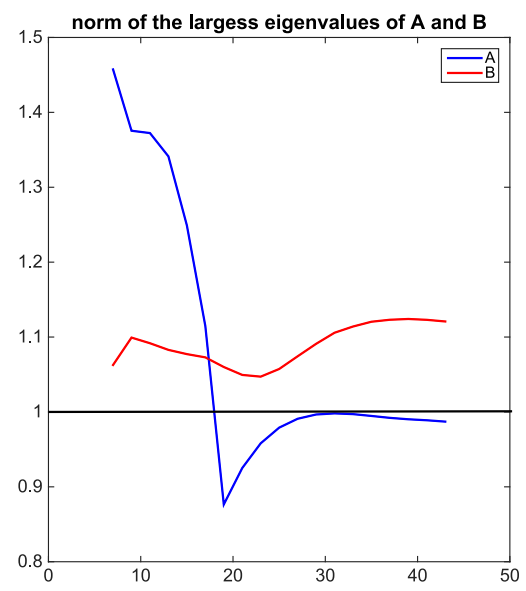

Fig. 6: Norms of the largest eigenvalue of matrices $A$ in blue and $B$ in red for hovering flight with various beat-wing frequencies.

\section{E. Effect of the wing morphology on stability of hovering flight}

In this section, we study the influence of the wings morphology on the stability of the hovering flight. In particular, we will see how the variations of the size of the wing defined by $C$ and $S_{p a}$, can affect the stability.

When the size of the wings increases, the lift increases so ensuring a hovering flight at lower flapping frequencies. The wings size has a significant effect on the stability of the flight. The stability of the hovering flight has been explored for simultaneous variations of its chord $C$ and its span $S_{p a}$ from $90 \%$ to $120 \%$ of the size of table I. This is illustrated in figure 7 which shows the contour of the largest eigenvalues of matrices $A$ and $B$ for hovering flight, as function of the size of the wing and of the beating frequency. Stable hovering flights are obtained for values of frequency and wing size such that the two largest eigenvalues are less that 1 and correspond to the white area of figure 7. For instance, increasing the wing size of $12 \%$ with respect to the initial design, leads to a stable hovering flight at $19 \mathrm{~Hz}$. The largest (non equal to 1) eigenvalues of the Jacobian matrix are in this case $\{0.8977,0.8977,0.9199,0.9084\}$.

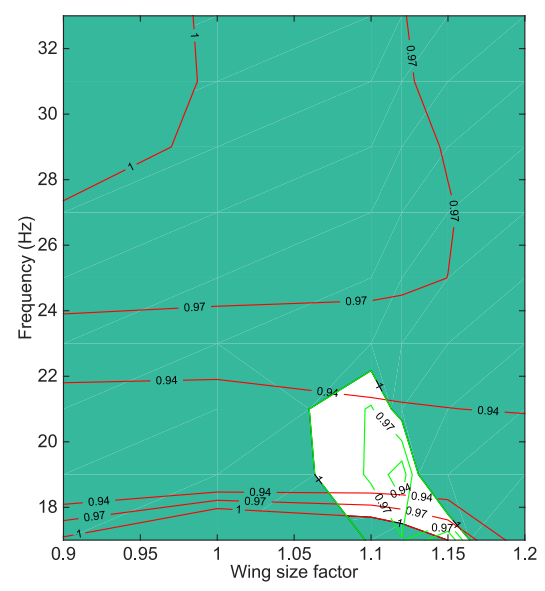

Fig. 7: Contour of the largest eigenvalues of matrices $A$ and $B$ for hovering flight, as function of the size of the wing and of the beating frequency. The white area corresponds to stable hovering flight.

\section{F. Illustration of the approach: A stable passive design}

As previously shown, some slight modifications of the initial design of table I allow obtaining stable hovering flight. To illustrate this we now present a stable hovering flight when the size ( $C$ and $S_{p a}$ ) of the wing is increased of $12 \%$. In this case, the above study of stability has shown how the hovering flight is locally stable. Further simulations, show that the basin of attraction of the limit cycle is quite large as this is shown by the motion performed when the MAV starts at rest with a flapping motion of $19 \mathrm{~Hz}$ and an amplitude of $1.0545 \mathrm{rad}$. In this case, the motion of the MAV converges to a stable hovering flight with a cyclic motion of the passive twist shown in the left part in figure 8 . On the right part of this figure, the motion of the center of the thorax is shown in the plane $\left(n_{e}, a_{e}\right)$ (the thorax does not move along axis $s_{e}$ ). After a transient motion, the MAV self-stabilizes its motion. The final position is not controlled since it depends on the initial conditions. Due to the invariance of the model with respect to the thorax position, the mechanical design cannot affect it as this is predicted by the corresponding eigenvalues of the Jacobian matrix which are all equal to 1. As a result, in this case, an external control loop should be added to perform a desired displacement of the MAV for example, by using a vision based sensor. A more exhaustive analysis of the hovering flight can be found in [9], where the influence of position of the wings along the thorax, and that of their stiffness are studied and combined to produce stable hovering flight without any high level feedback. This is in constrast to other approaches as [36], where the flight control of the Bat Bot (B2) robot is adapted at each flapping period to preserve stability. 

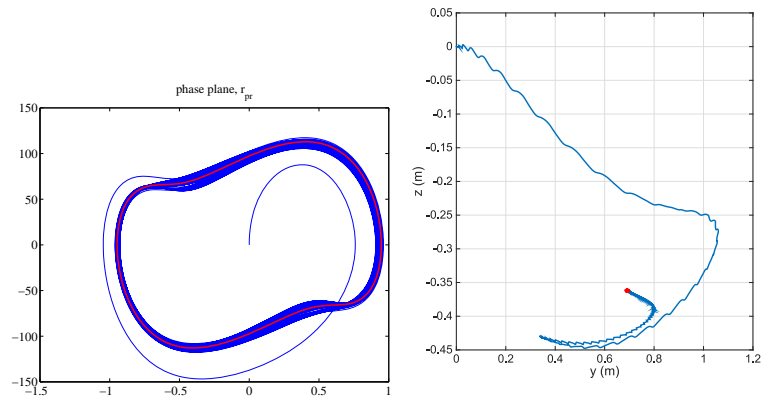

Fig. 8: The convergence of the motion of the MAV when starting from rest, it reaches hovering flight.

\section{THE WALL CLIMBING OF A GIBBON-LIKE ROBOT}

The arboreal apes, like the gibbon, evolve into the forest canopy, swaying from branch to branch in a motion called brachiation. This motion allows the ape to move in an efficient way. The key of success of such locomotion lies in the synchronization between the ape's body oscillation and its hand change. To study this locomotion mode, researchers from the University of Utah developed an original pendular climber, the ROCR, that can dynamically exploit gravity to perform efficient climbing gaits [35].

In this section, we consider a model of this robot as represented in figure 9. The considered robot has a tree-lik structure constituted of two rigid bodies. The upper body supports two grippers modelling the hands of the ape. The lower body is a pendulum whose oscillations stand for those of the ape's body and are assumed to be sinusoidal with fixed period and amplitude. Depending on the difference of phase between the pendulum oscillations and the change of supporting hand, this robot exhibits two different gaits (see figure 10) which can be stable or not. Remarkably, these two different gaits can be stable for the same control parameters, a situation in which the motion of the robot becomes unpredictable, since in this case, the gaits performed depend not only on the controller, but also on the initial state of the robot. The purpose of what follows consists in exploiting the robot's morphology to address this issue through what could be named a "morphological gait selection".

\section{A. Parametrization}

The orientation of the upper body with respect to the earth frame, is parameterized by $\theta$. The upper body is attached to the wall via two grippers ( $\mathrm{B}$ or $\mathrm{C}$ see figure 2 ) which are modeled as holonomic constraints. $r_{a}$ denotes the angle of the unique actuated joint, which connects the upper body and the pendulum. Regarding the geometric parameters, as depicted in figure 9, we denote by $a$, the distance between the center of the two grippers, $b$, the distance between the center of the frame $\mathcal{F}_{0}$ and the joint axis, and $c$, the length of the pendulum. The initial design is $a=0.1 \mathrm{~m}, b=0.02 \mathrm{~m}$, and $c=0.3 \mathrm{~m}$.

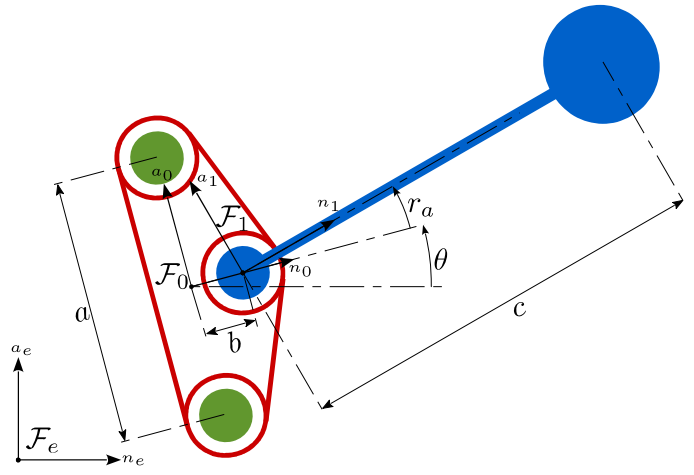

Fig. 9: Model of the wall climbing robot.

\section{B. Control law of the robot}

As mentioned before, a gibbon moves in the canopy by alternating its fixed hands rhythmically with the oscillations of its body. The swinging of the ape body is mimicked by imposing to the active joint the following cosine function:

$$
r_{a}^{d}(t)=A \cos \left(2 \pi \frac{t}{T}+\phi\right),
$$

where $A$ is the amplitude of the pendulum's oscillation and $T$, its period. The parameter $\phi$ defines the phase difference between the hand changes and the oscillation of the pendulum. To illustrate the role of morphology on the ROCR climbing, its hand changes are defined by switching the fixed gripper at each half period. Thus, the system is attached to the vertical wall through:

$$
\left\{\begin{array}{cc}
\text { the left gripper, } & \text { if } 0<t<T / 2 \\
\text { the right gripper, } & \text { if } T / 2<t<T
\end{array}\right.
$$

At $T / 2$ and $T$, the gripper in contact, changes from one hand to the other, and an impact instantaneously occurs in the locomotion model.

\section{Cyclic motion}

At each instant $t, x=\left(\theta, r_{a}, \dot{\theta}, \dot{r}_{a}\right)$ defines the full state of the robot and once controlled by (11), we can consider the evolution of its passive component $z_{p}=(\theta, \dot{\theta})^{\top}, \dot{r}_{a}$ and $r_{a}$ being expressed as functions of the control parameters $(A, T, \phi)$ according to the control law (11). In the rest of the section, the amplitude $A$ and the period $T$ are fixed to $60^{\circ}$ and $0.66 \mathrm{~s}$ respectively. Several cyclic motions can be obtained as function of $\phi$. Note that in this study, the displacement along $x$ and $y$ are not imposed, only the $\theta$ periodicity is considered. For a chosen $\phi$, the values of $\theta(0)$ and $\dot{\theta}(0)$ when the state crosses the Poincare section, i.e., when the left gripper attaches to the wall are determined as discussed in section III. After one period $T, \theta(T)=\theta(0)$ and $\dot{\theta}(T)=\dot{\theta}(0)$. Thus, with the motion of the actuated joint (11), and the switching condition (12), the robot returns in its initial state attached to the left gripper while being translated vertically of the net distance $-2 a \cos (\theta(0))$ which is indirectly governed by $\phi$ through the initial condition $\theta(0)$.

Remarkably, it can be observed in simulation that for any value of $\phi$, two cyclic motions of the climber can be obtained 
depending on their initial state denoted by $\left(\theta_{1}(0), \dot{\theta}_{1}(0)\right)$ and $\left(\theta_{2}(0), \dot{\theta}_{2}(0)\right)$ respectively. The first cyclic motion (defined by $\left(\theta_{1}(0), \dot{\theta}_{1}(0)\right)$ behaves as a pendulum, i.e. the tip mass of the pendulum moves under the gripper over one cycle. The second cyclic motion (defined by $\left(\theta_{2}(0), \dot{\theta}_{2}(0)\right)$ behaves as an inverted pendulum, i.e., the tip mass of the pendulum moves over the gripper while performing one cycle. These two gaits are illustrated in figure 10.

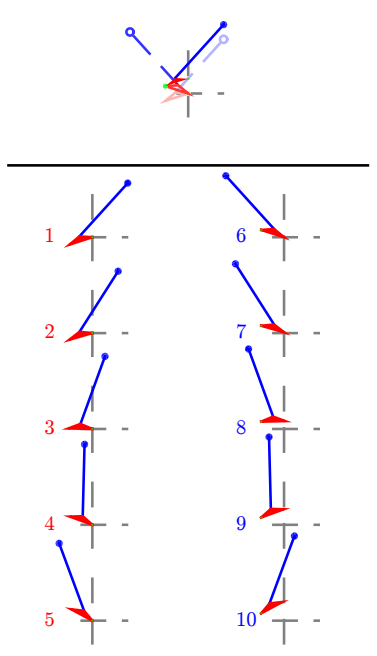

(a) inverted pendulum gait
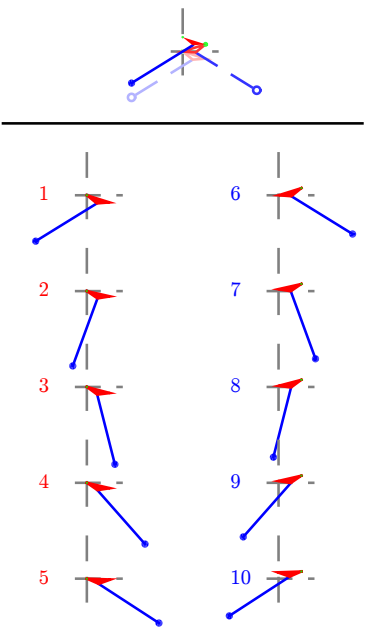

(b) pendulum gait
Fig. 10: Snapshots of two ascendant gaits, an "inverted pendulum"-gait and a "pendulum"-gait. The pictures numbered in red correspond to the left gripper configuration and those in blue, to the right gripper configuration. A collection of superimposed snapshots taken at the instants of the commutation of the grippers is presented at the top of the figures.

\section{Stability of the cyclic motions and Morphology}

For the initial morphology defined by $a, b, c$, the various cyclic gaits are illustrated in figure 11 through their initial passive state $(\dot{\theta}(0), \theta(0))$ as a function of the control variable $\phi$ (see figure 11a and 11b). The same figure displays the stability of each cyclic motion. The stable (respectively, unstable) gaits correspond to the blue (respectively, red) plots. These plots show that most of the pendulum gaits are stable, while most of the inverted pendulum gaits are unstable. However, for small value of $\phi$, the two gaits are unstable, as indicated by the red-colored area in figure 11. More remarkably around $\phi=\pi / 2$, the "pendulum" and "inverted pendulum"-gaits are both stable. These cases, which are indicated by the blue-colored area in figure 11, can be pathological for the control of the robot, since when a change of $\phi$ is desired, or in presence of perturbations, the final gaits can unpredictably converge to a pendulum or an inverted pendulum gait. In other terms, a same control $\phi$ can lead to the two different types of gait of the climber depending on its initial state. For example, it has been

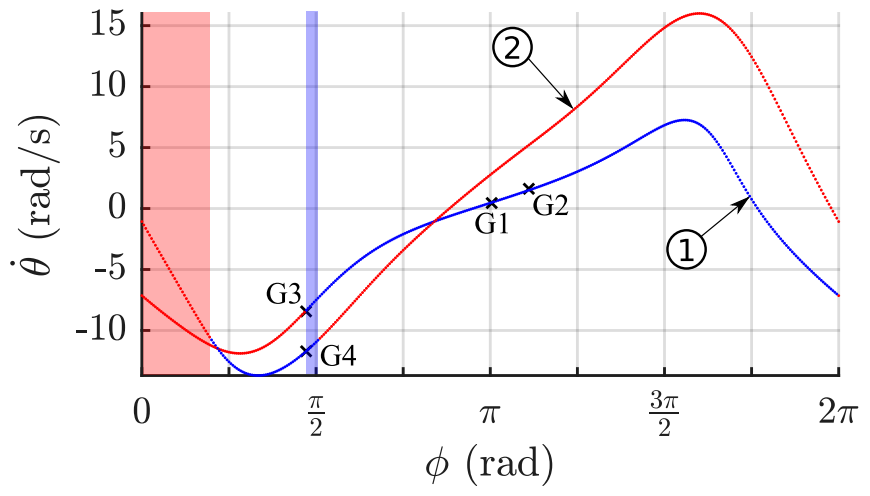

(a) $\dot{\theta}$ as a function of $\phi$

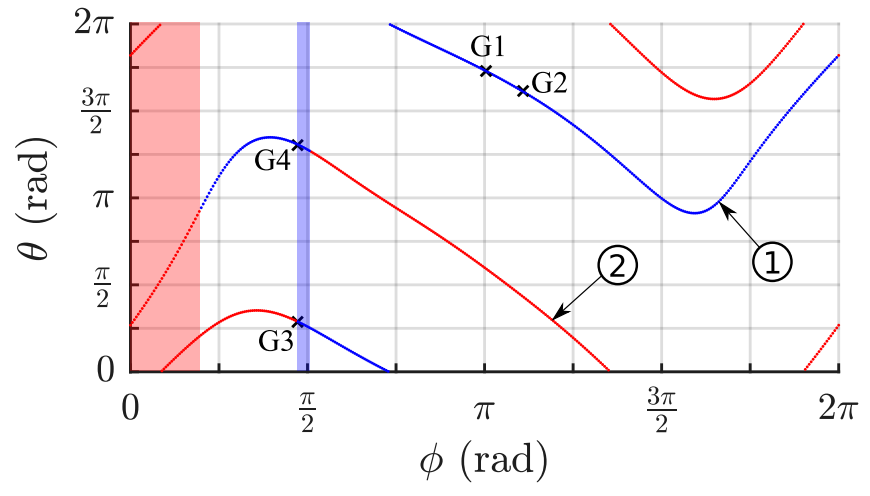

(b) $\theta$ as a function of $\phi$

Fig. 11: Initial state $\dot{\theta}(0), \theta(0)$ as function of $\phi$ corresponding to cyclic motions of the climber. The plots labeled 1 and 2 correspond to pendulum and inverted pendulum cyclic gaits respectively. The stable limit cycles are shown in blue and the unstable limit cycles in red. The red area represents the overlapping area of unstable gaits (i.e. the area where both cycles are unstable), while the blue one represents the overlapping of stable gaits.

observed in simulation the following facts. While starting with a stabilized gait denoted $G_{1}$ in figure 11, if the control law is suddenly switched to $\phi=1.48 \mathrm{rad}$ (corresponding to gait $G_{3}$ and $G_{4}$ ), the motion converges to the stable inverted pendulum cyclic motion $G_{4}$. In a similar way, when starting with a stabilized gait denoted $G_{2}$ in figure 11 , if the control law is again switched to $\phi=1.48 \mathrm{rad}$, the motion converges to the stable pendulum cyclic motion $G_{3}$. These cases illustrate the unpredictability due to the dependency on initial conditions.

To avoid this issue, we would like to have only one family of attractive (or stable) gaits for any value of $\phi$, while the second family of gaits should be unstable. For reasons of energy, our objective is to adapt the morphology of our climber in order to ensure stable pendulum gaits and unstable inverted pendulum gaits for any $\phi$. Figure 12 presents the effect of the parameters $a$ and $b$ on the ratio of stable gaits over the total number of gaits for each type of gaits ("pendulum" gaits in figure 10.a, and "inverted-pendulum"gaits in figure 10.b). 


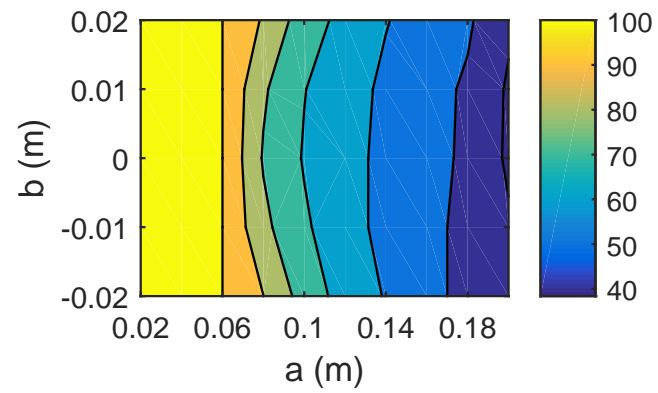

(a) Ratio of stable pendulum cycles

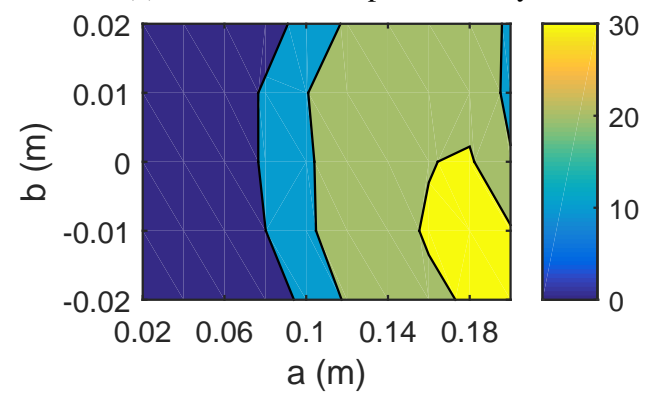

(b) Ratio of stable inverted pendulum

Fig. 12: Percentage of stable gaits with the pendulum configuration (Fig 12a) or the inverted pendulum configuration (Fig 12b) in function of the geometrical parameters $a$ and $b$.

While, the parameter $b$ has no significant influence on the ratio studied in figure 12 , we can notice that for $a<0.06$ $\mathrm{m}$, it is possible to completely remove the overlapping (blue and red) areas and to ensure that all the stable gaits are of pendulum type. Based on this remark, we can propose a set of new designs, e.g. $a=0.02 \mathrm{~m}$ with $b$ and $c$ unchanged. In this case, the cyclic gaits are shown as function of the control parameter $\phi$ in figure 13. These plots show that as expected, all the pendulum cyclic gaits are stable and all inverted pendulum gaits are unstable.
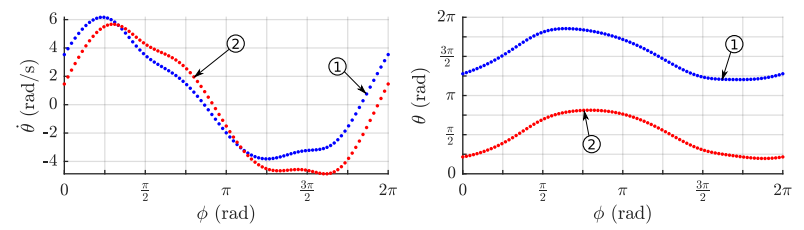

Fig. 13: Initial state $\dot{\theta}(0), \theta(0)$ as function of $\phi$ corresponding to cyclic motions of the climber. The plots labeled 1 and 2 correspond to pendulum and inverted pendulum cyclic gaits respectively. The stable limit cycles are shown in blue and the unstable limit cycles in red.

\section{Planar Walking in SagitTal Plane}

In this section, we consider the case of the planar walker of figure 2. The study of planar walking biped robots, as Rabbit, was the subject of many theoretical and experimental studies [2], [6], [26], [42], [48]. From the control point of view, the results presented are not new, but shed some new lights on the influence of the body morphology and the shape of the gait on a self stabilization of walking with a control law based on the tracking of virtual constraints. In this context, it has been shown that thanks to the concept of virtual constraints, a direct link between the stability of cyclic walking and the shape of the gait can be established. The purpose of what follows is to exploit this concept and to show that for a gait involving single supports and impacts where the leg that is in support takes off when the other touches the ground, a necessary condition to produce stable walking is as following. When the change of support occurs, the linear velocity of the center of mass just needs to be directed downward with respect to the ground. This simple condition for the control design is subtended by the good tuning of the walker body morphology to its walking gait. A summary of this semi-passive control strategy and the key milestones of its stability analysis are given hereafter.

According to remark 3 (see section III), the desired evolution of the actuated variables is defined as a function in the passive configuration $q_{p}=\theta$, i.e., it takes the form $r_{a}^{d}(\theta)$, where $\theta$ is defined as illustrated in figure 14. This allows removing the explicit time dependency of the configuration evolution, what is always possible when $\theta$ is a monotonic function in time. As a corollary, in all the following, the only role of the actuated torque $\tau_{a}$ will consist in tracking $r_{a}=r_{a}^{d}(\theta)$. In particular, the torque does not define the motion as a function of time. This is a crucial advantage since when a disturbance perturbs the motion of the robot, the low level torque controller will only have to maintain the shape-evolution of the robot, so avoiding the complex re-synchronization of the motion with an external clock.

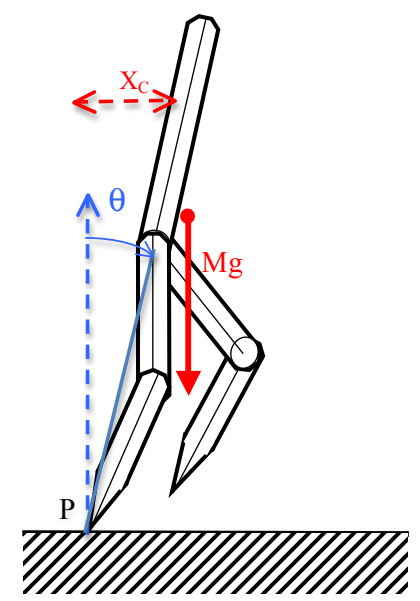

Fig. 14: The planar biped Rabbit and the definition of the angle of the virtual stance leg $\theta$.

Based on this approach, we are now going to show that the gravity entirely defines the time evolution of the robot's configuration. To that end, let us state the global balance dynamics applied to the robot around the passive external joint connecting the tip of the stance leg and the ground (located in $P$ in figure 14). We have:

$$
\dot{\sigma}=M g x_{c}(\theta)
$$


where $\sigma$ is the angular momentum of the robot around the point of contact $P$ in the sagittal plane, $M$ is the mass of the bipedal robot, $g$ is the acceleration of gravity, $x_{c}$ is the distance along the advance axis between the stance point foot and the CoM. A simple inspection of (13) shows that the global (net) rotation of the robot is only actuated by the gravity while the internal torques do not affect this global equilibrium. Based on this simple remark, one can infer the ballistic of one step. In details, the step starts with a given positive angular momentum, then during the first half part of the step, the CoM being behind $P$, the gravity first decreases the angular momentum of the robot. After this ascending phase, if the initial angular momentum is enough large, the CoM moves past $P$, and then the gravity increases the angular momentum so making fall the system until its swing leg touches the ground. Due to the adopted low-level control strategy $\left(r_{a}=r_{a}^{d}(\theta)\right)$, a coordination of the passive and active joints is naturally ensured, and all the velocity components are proportional to $\dot{\theta}$. As a result, the angular momentum takes the detailed form:

$$
\sigma=I(\theta) \dot{\theta}
$$

where $I(\theta)$ is the inertia momentum of the robot in the sagittal plane around $P$. This inertia varies as a function of the shape of the robot, itself defined as a function of $\theta$. This control strategy allows ensuring that the contact with the ground with both legs, which is here imposed by the geometrical constraint (2) (both legs touch the ground at a single instant), occurs for a known value of $\theta$, denoted $\theta^{+}$at the beginning of the step after impact, and $\theta^{-}$at the end of the step before impact. During the single support phase, the robot is feedback controlled with its low level control and behaves as a (semi) passive system under gravity with free rotation in the sagittal plane around $P$. As a consequence, one can define a "pseudo total energy" which is conserved along a step. Going further, it can be shown [6] that $\sigma^{2}$ plays the role of a "pseudo kinetic energy", while the change of its "pseudo potential" counterpart between successive single support phases (i.e., between the beginning and the end of a step), is the same from step to step. This basic fact is illustrated by the following conservation balance equation and by figure 15 .

$$
\left(\sigma_{k}^{-}\right)^{2}-\left(\sigma_{k}^{+}\right)^{2}=V=\text { cst. }
$$

In (15), $\sigma_{k}^{-}$and $\sigma_{k}^{+}$denote the angular momentum with respect to $P$, respectively at the end and beginning of step $k$.

The change of step being characterized by the change of support point, let us denote $d$ the step length, i.e., the distance between the two leg tips at the instant they touch the ground. During the impact, the angular momentum around the swing leg that touches the ground is conserved since the single external contact force is applied at that point. Hence, just before the impact, the angular momentum around the new contact point can be calculated using the transport rule of angular momentums as:

$$
\sigma_{k+1}^{+}=\sigma_{k}^{-}+M d v_{z}=\delta \sigma_{k}^{-},
$$

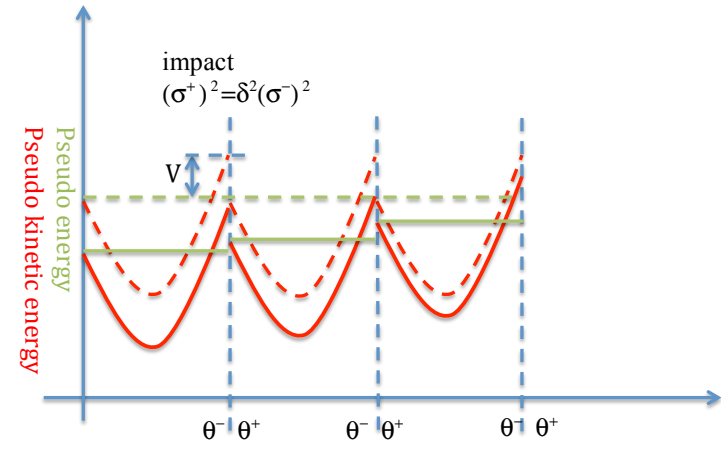

Fig. 15: A qualitative look at stability via the pseudo energy step after step. During each step, from $\theta^{+}$to $\theta^{-}$, the pseudo energy (in green) is constant and the pseudo kinematic energy (in red) evolves due to the gravity effect. At impacts, the pseudo kinematic energy is multiplied by $\delta^{2}$ while the pseudo potential energy returns to its initial level. The dotted line corresponds to a motion that starts on the cyclic motion, and the solid line to a motion that starts away from this cyclic case. The case $\delta<1$ is illustrated. The pseudo energy of the system converges to its cyclic value.

where, because the motion of all the internal joints are timecoordinated, the change of angular momentum is simply changed through a transition factor here denoted $\delta$. Equation (16) also shows that, since $d$ is positive, the angular momentum increases or decreases only depending on the sign of the vertical velocity of the center of mass $v_{z}$. In particular, a negative value of $v_{z}$, which corresponds to a velocity of the center of mass at the transition between two steps directed downward (toward the ground), produces a decrease of angular momentum indicated by a value of $\delta$ lower than 1 . Moreover, a cyclic motion is obtained when the variation of the "pseudo kinetic energy" exactly compensates that from the impact to recover the same value at the beginning of each two successive steps. As discussed in section III, the cyclic motion is characterized by a fixed point in the Poincaré section. In fact, a diffeomorphism in $S_{p a}$ between the passive variables $\left(q_{p}, \dot{q}_{p}\right)$ and $\left(\theta^{-}, \sigma^{-}\right)$can be defined. Thus, since $\theta^{-}$is constant, the cyclic motion is defined by two successive identical values of $\sigma^{-}$. According to equations (15) and (16), such a cyclic motion is characterized by:

$$
\left(\sigma_{*}^{-}\right)^{2}=\frac{V}{1-\delta^{2}}
$$

where the index $*$ stands for any step-number. After having defined such a cyclic motion, the next issue to address is to analyse its stability, i.e., we need to know what happens when the motion of the robot does not start on this cyclic motion, but close to it. To that end, let us assume that there is an initial error $\Delta_{k}$ on angular momentum $\sigma_{*}^{-}$or pseudo kinetic energy $\left(\sigma_{*}^{-}\right)^{2}$. Then according to the general framework of section IV we have $\left(\sigma_{k}^{-}\right)^{2}=\left(\sigma_{*}^{-}\right)^{2}+\Delta_{k}$. Remarking that after the impact, the pseudo kinetic energy becomes $\left(\sigma_{k+1}^{+}\right)^{2}=\delta^{2}\left(\left(\sigma_{*}^{-}\right)^{2}+\Delta_{k}\right)$, and due to the conservation of variation of pseudo energy (15) during the single support 
phase, after one step, the error on kinetic energy is $\delta^{2} \Delta_{k}$. Thus the decrease of the error on kinetic energy can only be performed if this error decreases at impact, i.e., if $\delta<1$. As a consequence, with the proposed low level controller, a stable walk will be obtained if $\delta<1$, i.e., if $v_{z}<0$. As regards the step length $d$, it can be seen in equation (16) that its value directly affects that of $\delta^{4}$, which characterizes the convergence to the cyclic motion. Going further, longer steps will induce faster convergence to the cyclic motion. Let us now infer the conditions of the realization of this condition and remark that since the angular momentum decreases at each change of support, the angular momentum has to increase during the single support phase. This is equivalent to say that we need to have $V>0$ or roughly speaking, that the mean value of $x_{c}$ during a single support needs to be positive (see figure 14). Another condition is that the angular momentum is always positive for all the single support phases. Finally these developments motivate the following concluding remark. The condition of stability being defined by an inequality $(\delta<1)$ it is intrinsically robust. In other words, if the model is not perfectly known, even based on an erroneous evaluation of $\delta$, the stability would be preserved. This theoretical expectation has been observed experimentally in [46]. To illustrate the walking of a planar robot using the proposed control law, a simulation of the robot Rabbit has been performed [7]. The reference path $r^{d}(\theta)$ is defined as a fourth order polynomial of $\theta$, and the corresponding stick diagram is shown in figure 16. The walking is quite human-like. As expected, due to the mass distribution, the center of mass is close to the hip and its velocity is directed downward at the end of the step. The convergence toward the cyclic motion is illustrated in figure 17 for the absolute angle of the trunk in its phase plane. Step after step the evolution of the joint converges to the cyclic motion.

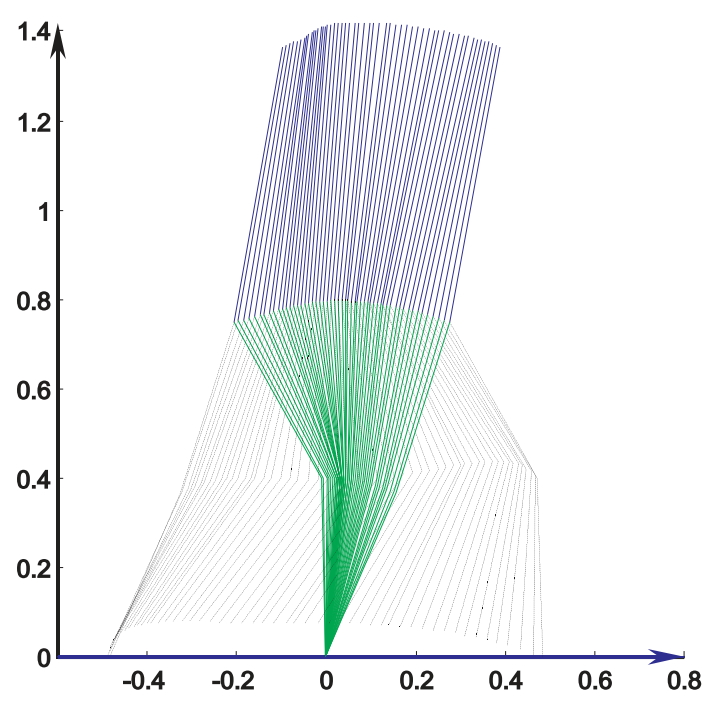

Fig. 16: Stick diagram of the walker.

\footnotetext{
${ }^{4}$ assuming that $V$ and $\frac{v_{z}}{\sigma_{k}^{-}}$are not modified
}

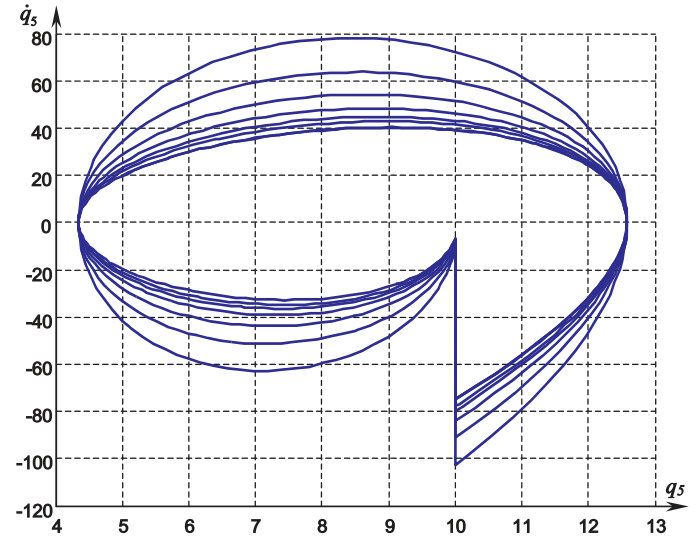

Fig. 17: Phase plane of the absolute angle of the walker's trunk.

\section{CONCLUSION}

To perform their locomotion gaits, animals exploit their physical surrounding to passively actuate some of their internal and external passive degrees of freedom through physical interactions in which the body morphology plays a key role. For instance, the soft body of a fish can be passively coupled with the vortices of an ambient flow to self-propel with no effort, while the wings of a moth passively twist to generate the lift required by hovering flight. On earth, another source of actuation is naturally provided by gravity that walkers and climbers exploit through ballistic phases to improve their locomotion performance. In this paper, a HZD-based methodological framework has been proposed to facilitate the Poincaré stability analysis of locomotion robot performing some expected gaits. By contrast to a full control based approach, where the control has to be adapted to a fixed robot's body, the design of gaits and the study of their stability is performed in parallel to the design of the body. This approach provides a systematic way to control locomotion cyclic motions with low-level feedback controllers. As a result, it allows avoiding complex computations based on exteroceptive feedbacks which are in this case outsourced to the body morphology. Our proposed methodology lies on a general modeling approach leading to a well suited control form in which the actuated variables have some prescribed evolutions imposed by low-level (proprioceptive) feedback loops, while the passive internal and external variables can interact passively with the physical surrounding of the robot. Such a control form is well suited to the study of locomotion since it preserves the mechanical properties of the system, as its inertia distribution, while reducing the dimension of the model to that of the state subspace of its passive variables. Based on this control-form, we used the concept of Poincaré return map, to define cyclic motions and to study their stability in a straightforward manner. Three cases have been used to illustrate the efficacy of our approach. The first is related to hovering flight of a MAV with soft wings, the second to pendular climbing and the third to bipedal walking. 
To encompass these examples in a same framework requires to consider different types of contact with the environment, which can be rigid or smooth, continuous or discontinuous. Moreover, the evolution of the actuated variables can be expressed as function of time or not, while the dimension of the passive state can vary from one to 16 , and that of the actuated variables, from one to 6 . When the robot has a small number of actuated joints, as this is the case of the MAV or the pendular climber, its behavior is largely affected by the morphology of its body, and especially by the position of the joints with respect to its main reference body. Thus the position of the wings of the MAV, or the position of the attachment points of the climber, strongly affects the stability of locomotion, and an appropriate design can be used to avoid high level control. These simple considerations do instantiate the concept of morphological computation, since in this case, the stability is simply ensured by the body morphology. When addressing systems with a larger number of actuated variables, as this is the case of walking, the concept of morphological computation has to be extended to the definition of the gaits, whose physical features in relation to the gravity can be considered as morphological characteristics. In this context, it has been shown that vertical oscillations of the mass center can allow self-stabilizing the locomotion of a planar biped. Beyond these particular examples, let us note that to numerically apply the concept of Poincare map to these systems, we have systematically exploited the symmetry properties of both the body, and the desired gaits motion. These properties, which are fundamentally based on a bi-lateral (right/left) symmetric morphology, along with the invariance of the dynamic model with respect to absolute translations and rotations around vertical axis (supported by gravity), can be extended to many other locomotion systems.

\section{REFERENCES}

[1] R. Mcneill Alexander. Principles of Animal Locomotion. Princeton University Press; New Ed, 2006.

[2] Y. Aoustin and A. M. Formal'sky. Control design for a biped: Reference trajectory based on driven angles as functions of the undriven angle. Journal of Computer and Systems Sciences International, 42(4), 2003.

[3] J. Bertram, A. Ruina, C. Cannon, Y. Chang, and M. Coleman. A pointmass model of gibbon locomotion. J. Exp. Biol, 202(19):2609-2617, 1999.

[4] R. Blickhan, A. Seyfarth, H. Geyer, S. Grimmer, H. Wagner, and M. Günther. Intelligence by mechanics. Philosophical Transactions of the Royal Society of London A: Mathematical, Physical and Engineering Sciences, 365(1850):199-220, 2007.

[5] C. Canudas-de Wit. On the concept of virtual constraints as a tool for walking robot control and balancing. Annual Reviews in Control, 28(2): 157 - 166, 2004.

[6] C. Chevallereau, G. Abba, Y. Aoustin, F. Plestan, E. R. Westervelt, C. Canudas-de-Wit, and J. W. Grizzle. RABBIT: A testbed for advanced control theory. IEEE Contr. Syst. Mag., 23(5):57-79, October 2003.

[7] C. Chevallereau, A. M. Formal'sky, and D. Djoudi. Tracking of a joint path for the walking of an underactuated biped. Robotica, 22:15-28, 2004.

[8] C. Chevallereau, J.W. Grizzle, and C-L. Shih. Asymptotically stable walking of a five-link underactuated 3D bipedal robot. IEEE Trans. Robot., 25(1):37-50, 2009.
[9] C. Chevallereau, M. Porez, and F. Boyer. Computational morphology for a soft micro air vehicle in hovering flight. In 2014 IEEE/RSJ International Conference on Intelligent Robots and Systems, pages 3404-3410, Sept 2014.

[10] M. Coleman. A Stability Study of a Three-Dimensional PassiveDynamic Model of Human Gait. PhD thesis, Cornell University, 2006.

[11] S. H. Collins, M. Wisse, and A. Ruina. A 3-D passive dynamic walking robot with two legs and knees. Int. J. Robot. Res., 20:607-615, 2001.

[12] X. Da, O. Harib, R. Hartley, B. Griffin, and J. W. Grizzle. From 2d design of underactuated bipedal gaits to $3 \mathrm{~d}$ implementation: Walking with speed tracking. IEEE Access, 4:3469-3478, 2016.

[13] M. H. Dickinson, F.-O. Lehmann, and S. P. Sane. Wing rotation and the aerodynamic basis of insect flight. Science, 284:1954-1960, 1999.

[14] M.H. Dickinson, C.T. Farley, R.J. Full, M.A. Koehl, R. Kram, and $\mathrm{S}$ Lehman. How animals move: An integrative view. Science, 288(5463): 100-106, 2000.

[15] R. J. Full and C. T. Farley. Musculoskeletal dynamics in rhythmic systems - a comparative approach to legged locomotion. In J. M. Winters and P. E. Crago, editors, Biomech. Neural Contr. Posture Movement. Springer-Verlag, New York, 2000.

[16] M. W. Gomes and A. L. Ruina. A five-link 2d brachiating ape model with life-like zero-energy-cost motions. Journal of Theoretical Biology, 237(3):265 - 278, 2005.

[17] J.W. Grizzle, C. Chevallereau, R.W. Sinnet, and A.D. Ames. Models, feedback control, and open problems of $3 \mathrm{~d}$ bipedal robotic walking. Automatica, 2014.

[18] J. Guckenheimer and P. Holmes. Nonlinear Oscillations, Dynamical Systems, and Bifurcations of Vector Fields. volume 42 of Applied Mathematical. Springer-Verlag, New York, 1996.

[19] T. L. Hedrick and T. L. Daniel. Flight control in the hawkmoth manduca sexta: the inverse problem of hovering. Journal of Experimental Biology, 209(16):3114-3130, 2006.

[20] K. Hirai, M. Hirose, Y. Haikawa, and T. Takenaka. The development of Honda humanod robot. In Proc. 1998 IEEE Int. Conf. Robot. Autom. (ICRA), volume 2, pages 1321-1326, Leuven, May 1998.

[21] Y. Hürmüzlü and D. B. Marghitu. Rigid body collisions of planar kinematic chains with multiple contact points. Int. J. Robot. Res., 13(1):82-92, 1994.

[22] Auke J Ijspeert. Biorobotics: Using robots to emulate and investigate agile locomotion. Science, 346(196), 2014.

[23] Christine Chevallereau Jessy Grizzle. Handbook of Humanoid Robotics, volume HUMANOID CONTROL (HC), chapter Virtual Constraints and Hybrid Zero Dynamics for Realizing Underactuated Bipedal Locomotion. Springer, 2016.

[24] J. C. Liao. A review of fish swimming mechanics and behaviour in altered flows. Philosoph. Trans B, 362(1487):1973-1993, 2007.

[25] R. Madangopal, Z.A. Khan, and S.K. Agrawal. Biologically inspired design of small flapping wing air vehicles using four-bar mechanisms and quasi-steady aerodynamics. Journal of Mechanical Design, 127:809-816, 2005.

[26] S. Miossec and Y. Aoustin. A simplified stability study for a biped walk with under and over actuated phases. International Journal of Robotics Research, 24(7):537-551, 2005.

[27] V. C. Müller and M. Hoffmann. What is morphological computation? on how the body contributes to cognition and control. Artificial Life, 23(1):1-24, February 2017.

[28] J. Nakanishi, T. Fukuda, and D. E. Koditschek. Analytical approach to studies of two-link brachiating robot control. Journal of the Robotics Society of Japan, 16(3):361-368, 1998.

[29] J. Nakanishi, T. Fukuda, and D. E. Koditschek. A brachiating robot controller. IEEE Transactions on Robotics and Automation, 16(2):109123, Apr 2000.

[30] T. S. Parker and L. O. Chua. Practical Numerical Algorithms for Chaotic Systems. Springer-Verlag, New York, 1989.

[31] R. Pfeifer and J. Bongard. How the body shapes the way we think: a new view of intelligence. MIT press, 2006.

[32] R. Pfeifer, M. Lungarella, and F. Iida. Self-organization, embodiment, and biologically inspired robotics. Science, 318(5853):1088-1093, 2007.

[33] M. Porez, F. Boyer, and A. Belkhiri. A hybrid dynamic model for bio-inspired robots with soft appendages - application to a bio-inspired flexible flapping-wing micro air vehicle. In IEEE ICRA, June 2014.

[34] M. Porez, F. Boyer, and A. Belkhiri. A hybrid dynamic model for bioinspired soft robots - application to a flapping-wing micro air vehicle. 
In 2014 IEEE International Conference on Robotics and Automation (ICRA), pages 3556-3563, May 2014.

[35] W. R. Provancher, S. I. Jensen-Segal, and M. A. Fehlberg. Rocr: An energy-efficient dynamic wall-climbing robot. IEEE/ASME Transactions on Mechatronics, 16(5):897-906, Oct 2011.

[36] A. Ramezani, S.-J. Chung, and S. Hutchinson. A biomimetic robotic platform to study flight specializations of bats. Science Robotics, 2(3), 2017.

[37] S. Revzen, S. Burden, D. Koditschek, and S. S. Sastry. Pinned equilibria provide robustly stable multilegged locomotion. Dynamic Walking, 2013.

[38] T.J. Roberts and E. Azizi. Flexible mechanisms: The diverse roles of biological springs in vertebrate movement. Journal of Experimental Biology, 214(3):353-361, 2011.

[39] S. I. Jensen Segal, S. Virost, and W. R. Provancher. Rocr: Dynamic vertical wall climbing with a pendular two-link mass-shifting robot. In IEEE Int. Conf. Robot. Autom, pages 3040-3045, 2008.

[40] C-L. Shih, J.W. Grizzle, and C. Chevallereau. From stable walking to steering of a $3 \mathrm{D}$ bipedal robot with passive point feet. Robotica, 30(7):1119-1130, December 2012. available at http://www.eecs.umich.edu/ grizzle.

[41] M. W. Spong and F. Bullo. Controlled symmetries and passive walking. IEEE Trans. Autom. Contr., 50(7):1025-1031, 2005.

[42] K. Sreenath, H.-W. Park, I. Poulakakis, and J. W. Grizzle. A compliant hybrid zero dynamics controller for stable, efficient and fast bipedal walking on MABEL. Int. J. Robot. Res., 30(9):1170-1193, 2011.

[43] R. Tedrake, T.W. Zhang, and H.S. Seung. Learning to walk in 20 minutes. In Proc. Fourteenth Yale Workshop on Adaptive and Learning Systems, Yale University, New Haven, CT, 2005.

[44] D. Tlalolini Romero, C. Chevallereau, and Y. Aoustin. Comparison of different gaits with rotation of the feet for a planar biped. Robotics and Autonomous Systems, 57(4):371-383, April 2009.

[45] T. Wang, C. Chevallereau, and C.F. Rengifo. Walking and steering control for a 3D biped robot considering ground contact and stability. Robot. Auton. Syst., 60(7):962-977, 2012.

[46] E. R. Westervelt, G. Buche, and J. W. Grizzle. Experimental validation of a framework for the design of controllers that induce stable walking in planar bipeds. Int. J. Robot. Res., 24(6):559-582, 2004.

[47] E. R. Westervelt, J. W. Grizzle, and C. Canudas-de-Wit. Switching and PI control of walking motions of planar biped walkers. IEEE Trans. Autom. Contr., 48(2):308-312, February 2003.

[48] E. R. Westervelt, J. W. Grizzle, C. Chevallereau, J.-H. Choi, and B. Morris. Feedback Control of Dynamic Bipedal Robot Locomotion. CRC Press, Boca Raton, 2007.

[49] E. R. Westervelt, J. W. Grizzle, and D. E. Koditschek. Hybrid zero dynamics of planar biped walkers. IEEE Trans. Autom. Contr., 48(1):42-56, 2003.

[50] A. Willmott and C. Ellington. The mechanics of flight in the hawkmoth manduca sexta. i. kinematics of hovering and forward flight. Journal of Experimental Biology, 200:2705-2722, 1997. 